\title{
Gamma-ray bursts detected from BATSE DISCLA data
}

\author{
M. Schmidt \\ California Institute of Technology, Pasadena, CA 91125, U.S.A \\ e-mail: mxs@deimos.caltech.edu
}

Received January 21; accepted February 28, 1999

\begin{abstract}
We have searched for gamma-ray bursts (GRB) in the BATSE DISCLA data covering the period TJD 8365-10528. We employ an algorithm that uses the background both before and after the onset of the burst, and that requires an excess of at least $5 \sigma$ over background in the energy range $50-300 \mathrm{keV}$. For the initial set of 7536 triggers, we find strong concentrations at given geographic locations of the satellite. After excluding these geographic areas, we are left with 4485 triggers. We exclude triggers close in position to solar flares, CygX-1, and Nova Persei 1992, when active as well as soft bursts close to the sun. We accept 1018 triggers that occur within 230 s of a GRB listed in the BATSE catalog, and upon visual inspection classify 881 triggers as magnetospheric events and 404 as GRBs. The final sample of 1422 GRBs represents effectively 2.0 years of isotropic exposure, for an annual rate of 710 GRBs per year.
\end{abstract}

Key words: gamma-ray bursts

\section{Introduction}

The Burst and Transient Source Experiment (BATSE) on board the Compton Gamma Ray Observatory has been very effective in recording gamma-ray bursts (GRB). Soon after the observatory's launch on April 19, 1991, the results obtained with BATSE showed convincingly that the sky distribution of GRBs was isotropic (Meegan et al. 1992a) and that the line-of-sight distribution was incompatible with a homogeneous distribution in euclidean space (Meegan et al. 1992b). The proposition based on these findings that the distance scale of GRBs is cosmological (Paczynski 1992) was eventually confirmed by the observation of a large redshift (Metzger 1997).

The GRB data from BATSE have been published primarily in a succession of catalogs (cf. Meegan et al. 1997). These data are based on an on-board trigger, which acts

Send offprint requests to: M. Schmidt when certain conditions are fulfilled. Usually these require that the counts in the energy range $50-300 \mathrm{keV}$ exceed the background by $5.5 \sigma$ on a time scale of 64 , 256 or $1024 \mathrm{msec}$ in at least two of the eight detectors. Variations of these criteria, in the channels and the signalto-noise limits used, have been in effect at various times (cf. Meegan et al. 1997).

BATSE produces data in various modes in archival form. The DISCLA data provide a continuous record of the counts in channels $1,2,3$, and 4 for the eight detectors on a time scale of $1024 \mathrm{msec}$. The DISCLA data allow the detection of GRBs a posteriori in a way similar to the on-board trigger (except for the availability of only one time scale).

There are distinct advantages to searching for GRBs in archival data: the detection parameters can be set independently of those that are hard-wired in the on-board trigger mechanism; the search can be repeated with a different detection algorithm; each search can be carried out on the full data set, etc. A search for GRBs not detected by the on-board BATSE trigger has been conducted by Kommers et al. (1997).

We have used the DISCLA data to search for GRBs in the time period TJD 8365 - 10528. In the following sections we describe the search, the classification of the triggers, and the derivation of $\left\langle V / V_{\max }>\right.$ of the resulting sample.

\section{The search}

We have tried to automate the search procedure as much as possible, for the sake of both speed and consistency. This will allow simulations to be carried out to investigate various scientific problems. Our search has some similarities to that carried out by the on-board BATSE trigger: using the counts in channels $2+3$ covering the energy range $50-300 \mathrm{keV}$; averaging background counts over $17.408 \mathrm{~s}$; and requiring that two modules see a minimum excess over background. We differ, however, in the evaluation of the background as explained below. 


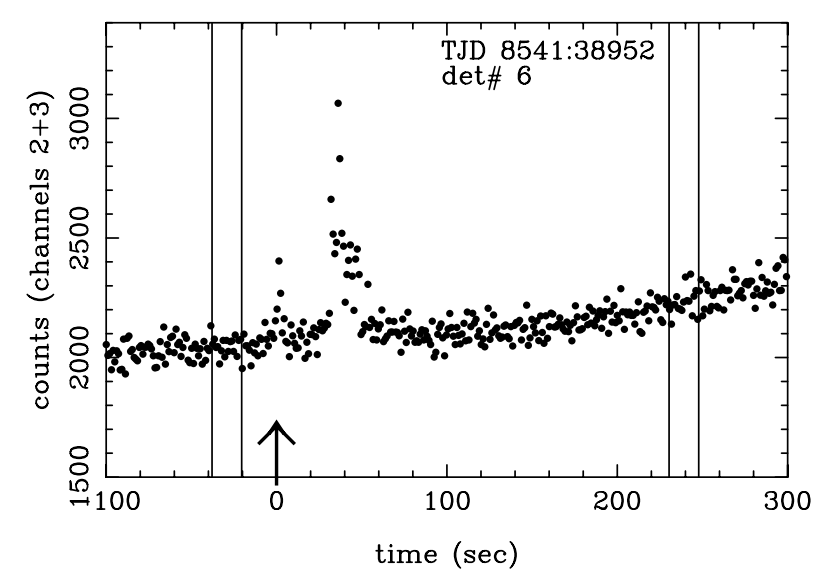

Fig. 1. The test for the presence of a burst at time 0.0 involves two intervals of $17.408 \mathrm{~s}$ in which the background is derived, one ending at time $-20.48 \mathrm{~s}$, the other beginning at time $230.4 \mathrm{~s}$, see text

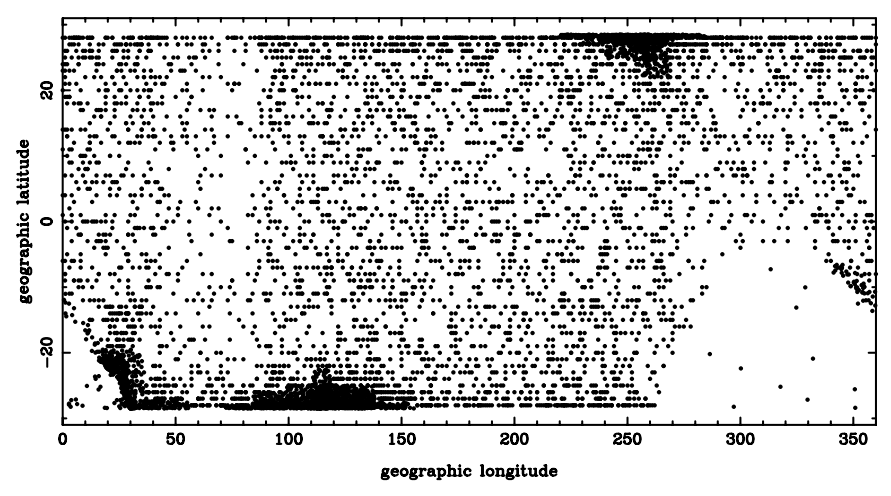

Fig. 2. Geographic positions of the Compton satellite during 7536 triggers. The geographic areas in the denser parts of the plot were excluded from the search, eliminating 3051 triggers

In the search conducted by the on-board BATSE trigger on the 1024 msec time scale, the background is derived over a given stretch of $17.408 \mathrm{~s}$ and the trigger test is carried out for the $1024 \mathrm{msec}$ bin following that stretch. The same background stretch is used for the next sixteen $1024 \mathrm{msec}$ bins, so that there is a separation between the end of the background stretch and the test bin of $0-16.384 \mathrm{~s}$.

We use a fixed separation between the background stretch and the test bin, and also introduce a second background stretch allowing a linear interpolation of the background for the duration of the GRB, cf. Fig. 1. For a small or zero separation between background stretch and test bin, slowly rising GRBs may escape detection (Higdon \& Lingenfelter 1996). If we increase the separation, more slowly rising GRBs can be detected, but then we find that the number of false triggers caused by higher-order variations in the background increases substantially. In the current search, we used a separation of $20.48 \mathrm{~s}$, and placed the beginning of the second background stretch $230.4 \mathrm{~s}$

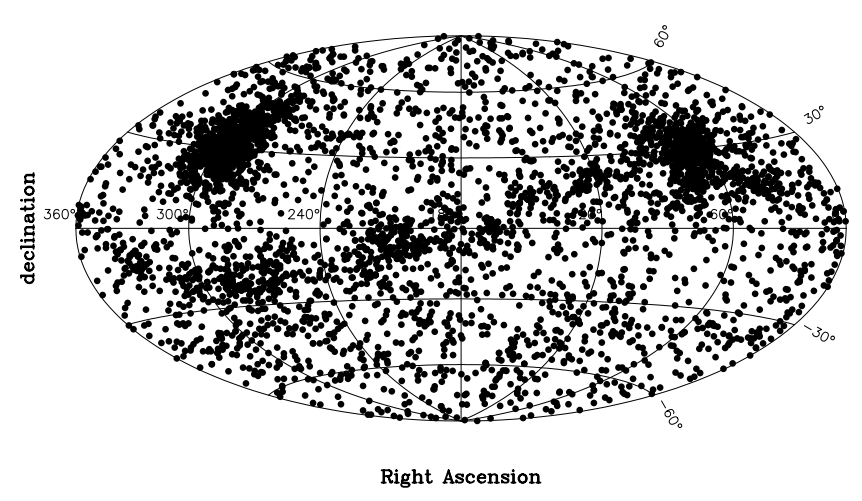

Fig. 3. Equatorial coordinates of 4485 triggers. The effects of CygX-1, Nova Persei 1992, and solar flares along the ecliptic are clearly seen. The remaining background triggers are partly magnetospheric events, and partly GRBs

after the test bin, as shown in Fig. 1. Following a burst, we disabled the trigger mechanism for $230.4 \mathrm{~s}$.

Artifacts or defects in the data will lead to false triggers. We have systematically searched for gaps in the data, and for constant output numbers (usually zeroes), and, from quality data provided by the BATSE project, for the occurrence of checksum errors. In each case, we set up a time window of exclusion around the defect, so that the automatic application of our search algorithm will not lead to a trigger. Considerable time is lost: the total number of our exclusion windows is over 151000 , of which some overlap.

We adopted a limiting $S / N$ ratio for detection in two detectors of 5.0. Our search of the time period TJD 8365-10528 with the criteria described above yielded 7536 triggers. The geographic coordinates of the observatory at the time of trigger are plotted in Fig. 2. Besides some areas near the South Atlantic Anomaly, there are clear concentrations in the south over W. Australia and in the north over Mexico and Texas. The southern concentration has been discussed by Horack et al. (1992). Since these concentrations have nothing to do with cosmic GRBs, we outlined geographic exclusion regions to avoid most of these triggers. With these exclusions in place, we are left with 4485 triggers, which form the basis for classification and discussion.

\section{Classification of triggers}

For each of the 4485 triggers, we derived sky positions on the assumption that they corresponded to the emission from a point source. We used the response function for the eight detectors (cf. Pendleton et al. 1995), and assumed a Band et al. (1993) type spectrum with $\alpha=-1$, $\beta=-2$, and break energy $E_{0}=150 \mathrm{keV}$. The positions were derived by testing around 10,000 positions on the sky and minimizing $\chi^{2}$ of the counts for all eight detectors. We ignored the effects of Compton scattering on the Earth's atmosphere or the spacecraft. Figure 3 shows the celestial coordinates of all 4485 triggers. 
Table 1. Classification of 4485 triggers

\begin{tabular}{lrr}
\hline Description & reject & accept \\
\hline Within 23 deg of sun, when active & 963 & \\
Within 23 deg of CygX-1, when active & 827 & \\
Within 23 deg of Nova Per 1992, when active & 418 & \\
Within 230 s of BATSE-listed GRB & & 1018 \\
Profile inspected: accepted as GRB & & 389 \\
Profile inspected: probably a GRB & & 15 \\
Profile inspected: rejected & 881 & \\
Spectrum very soft, near sun: rejected & 44 & \\
\hline
\end{tabular}

Total number of GRBs in DISCLA sample:

It is clear from an inspection of Fig. 3 that at least four types of triggers are present. Both CygX-1 and Nova Persei 1992 are prominent. The sun is clearly shown by solar flares along the ecliptic. There is a fourth component which appears more or less isotropic.

Plots of the number of triggers versus time close to the sky positions of CygX-1 and of Nova Persei 1992 show that both were detected during well-defined periods of activity. We used our positions of Nova Persei 1992, which totally dominated activity in its part of the sky for around 60 days, to evaluate the distribution of position errors. Based on these results, we eliminated from consideration all triggers within $23 \mathrm{deg}$ of the sun, of CygX-1, and of Nova Persei 1992 during the periods that these sources were active, see Table 1 .

We accept as GRBs all triggers whose onset was within $230.4 \mathrm{~s}$ of those listed for GRBs in the BATSE catalog (cf. Meegan et al. 1997 and the World Wide Web version maintained by the BATSE Burst team). For the remaining triggers, we inspected each of their time profiles from -300 to +400 s relative to the trigger time. In our judgment of the nature of these events we were guided by descriptions by the BATSE team of magnetospheric events (cf. Fishman et al. 1992; Horack et al. 1992), by the value of $\chi^{2}$ of the solution, by the dispersion of the positions obtained during each second that the burst was brighter than the limiting flux, and by a derivation of the angular motion of the source (which for a GRB should be consistent with zero). In the process, we noticed that 44 of the remaining triggers had spectra softer than any listed in the BATSE catalog, and that they were all within 35 deg of the sun. On the basis of this evidence, we rejected these as GRBs. The results of this excercise are shown in Table 1. We accepted 404 GRBs that are not listed in the BATSE catalog.

There are 130 GRBs listed in the BATSE catalog that we have not detected, while as stated we find 404 GRBs that are not in the catalog. The difference in content between the BATSE catalog and our sample is partly a consequence of differences in the de-activation of the trigger following a burst or around a defect in the data, and partly caused by statistical differences in the different backgrounds used.

Based on the entire search procedure, including the time windows of exclusion set up around bad data, the exclusion of particular geographic areas and of some sky areas in the classification procedure, and the part of the sky occulted by the Earth, we estimate that the sample of 1422 GRBs represents effectively 2.0 years of isotropic exposure by BATSE, for an annual detection rate of 710 GRBs per year.

\section{Derivation of $<V / V_{\max }>$}

The most important property of the GRBs in the sample is the euclidean value of $<V / V_{\max }>$. We will use its value to derive a distance scale for GRBs in a subsequent communication.

In most evaluations of $V / V_{\max }$ for individual GRBs, it has been assumed that it equals $\left(C_{\max } / C_{\min }\right)^{-3 / 2}$ where $C_{\min }$ is the minimum detectable count rate and $C_{\max }$ is the count rate at the maximum amplitude of the burst. This may not be correct. If we remove the source to the largest distance at which it is just still detectable, it is likely that the detection of the burst will occur later, and therefore that $C_{\min }$ will include some burst signal. Also, in some cases the part of the burst containing $C_{\max }$ will not cause a trigger when we remove the burst. Due to these inescapable effects, $V / V_{\max }$ will be larger than follows from the values of $C_{\max }$ and $C_{\min }$ at detection. This situation was encountered by Higdon \& Schmidt (1990) in their discussion of GRBs from the Venera 11 and 12 KONUS experiments.

We have derived the euclidean value of $V / V_{\max }$ for each GRB as follows. Upon detection, we derive the burst time profile by subtracting the background (interpolated between the two background stretches, see Fig. 1) from the counts. We multiply this original time profile by a factor of $X$ and add it to the background. Next we apply the search algorithm to see whether we detect a burst. If so, we repeat the process with a smaller value of $X$, until we do not detect a burst anymore. This represents the situation (as best we can) when the source has been removed in distance by a factor $X^{-1 / 2}$ in euclidean space. Therefore $V / V_{\max }$ for the source is $X^{3 / 2}$. Application of this process to all bursts in the sample produces $\left\langle V / V_{\max }>=0.334 \pm 0.008\right.$.

Acknowledgements. During the many years in which this work came to fruition, I have had much appreciated help from or discussions with D. Chakrabarty, M. Finger, G. Fishman, J. Gunn, J. Higdon, J. Horack, C. Meegan, T. Prince, and B. Vaughan.

\section{References}

Band D.L., et al., 1993, ApJ 413, 281

Fishman G.J., Meegan C.A., Wilson R.B., et al., 1992, in: AIP Conf. Proc. 265, Gamma-Ray Bursts, Paciesas W.S., Fishman G.J. (eds.). AIP, New York, p. 13 
Higdon J.C., Lingenfelter R.E., 1996, in: AIP Conf. Proc. 384, Gamma-Ray Bursts, Kouveliotou C., Briggs M.F. Fishman G.J. (eds.). AIP, New York, p. 402

Higdon J.C., Schmidt M., 1990, ApJ 355, 13

Kommers J.M., Lewin W.H.G., Kouveliotou C., et al., 1997, ApJ 491, 704

Meegan C.A., Fishman G.J., Wilson R.B., et al., 1992a, Nat 355,143

Meegan C.A., Fishman G.J., Wilson R.B., et al., 1992b, in: AIP Conf. Proc. 265, Gamma-Ray Bursts, Paciesas W.S.,
Fishman G.J. (eds.). AIP, New York, p. 61

Meegan C.A., Paciesas W.S., Pendleton G.N., et al., 1997, in: AIP Conf. Proc. 428, Gamma-Ray Bursts, Meegan C.A., Preece R.D., Koshut T.M. (eds.). AIP, New York, p. 3

Metzger M., et al., 1997, Nat 387, 878

Paczynski B., 1992, in: AIP Conf. Proc. 265, Gamma-Ray Bursts, Paciesas W.S., Fishman G.J. (eds.). AIP, New York, p. 144

Pendleton G.N., Paciesas W.S., Mallozzi R.S., et al., 1995, Nucl. Instrum. Methods Phys. Res. A 364, 567 